\title{
Alteraciones de la pragmática de la comunicación después de un traumatismo craneoencefálico
}

\author{
J.M. Muñoz-Céspedes ${ }^{\text {a,b }}$, N. Melle ${ }^{\text {a,c }}$
}

\section{PRAGMATIC IMPAIRMENTS FOLLOWING TRAUMATIC BRAIN INJURY}

\begin{abstract}
Summary. Objective. To describe how cognitive impairments contribute to the loss of communicative competence after traumatic brain injury (TBI), what instruments can be used to evaluate the pragmatic skills and which therapeutic approaches may be used to improve or compensate for this deficit. Discussion. We present a detailed bibliographic review on the topic that shows how certain functions (namely, memory, attention and executive functions) interact with communication skills, both expressive and comprehensive. The pragmatic approaches for cognitive-communicative TBI impairments are allow to count typical difficulties that are described (difficulty with topic selection, turn-taking initiation, ability to respond or give indirect requests, ability to meet the informational needs of the listener. appropriateness of utterances within conversation, etc). Next a general outline of the assessment and treatment of is provided, including several strategies based on recovery and functional adaptation and compensation. Conclusions. Given the huge influence of communicative skills on social and vocational integration, it is crucial to obtain a better understanding of the interaction between cognitive functions and communicative skills. Therefore, we need to devise assessment protocols specifically designed for Spanish speakers as well as new therapeutic approaches to increase the life quality of this population. The specific approaches to improve narrative, procedural and conversational discourse must divide from the components of the pragmatic competence and promote the cooperative participation of the teamwork who attend to the patient. [REV NEUROL 2004; 38: 852-9]
\end{abstract}

Key words. Cognitive functions. Neuropsychological assessment. Neuropsychological rehabilitation. Pragmatic. Right hemisphere. Traumatic brain injury.

\section{INTRODUCCIÓN}

Los traumatismos craneoencefálicos (TCE) traen consigo una serie de manifestaciones clínicas que son perceptibles ya desde las primeras fases tras el accidente. Entre esas consecuencias se encuentran las limitaciones físicas y las alteraciones de la memoria, la atención, las funciones ejecutivas (FE), el lenguaje, etc., que pueden diagnosticarse por medio de la administración de las pruebas adecuadas. Si bien estos factores van a determinar el tipo de vida del paciente después de la lesión, cabe destacar que existe otro factor que ejerce un impacto significativo, pero a largo plazo, sobre la calidad de vida y la integración social y laboral de la persona que ha sufrido un TCE.

Este último factor hace referencia a la pérdida de habilidades pragmáticas (HP), que participa en el deterioro de la red de amistades previa, el incremento del riesgo de divorcio, la tendencia al aislamiento social y la incapacidad para la reintegración laboral [1,2].

El interés por estas habilidades tuvo sus orígenes en los años 70 , cuando los instrumentos tradicionales para la evaluación de la afasia que se centraban en los aspectos puramente lingüísticos no eran capaces de reflejar de forma precisa las alteraciones comunicativas que se observaban en los pacientes que habían sufrido un TCE. Esto llevó al planteamiento de la existencia de un tipo de afasia, que Sarno [3] denominó 'afasia subclínica’. A lo largo de los años 80 se produjo un giro en la expli-

Recibido: 26.09.03. Aceptado tras revisión externa sin modificaciones: 15.01.04.

${ }^{a}$ Unidad de Daño Cerebral. Hospital Beata María Ana. ${ }^{b}$ Departamento de Psicología Básica (Procesos Cognitivos). Universidad Complutense de Madrid. ${ }^{c}$ Facultad de Psicología. Centro de Estudios Universitarios. San Pablo-CEU. Madrid, España.

Correspondencia: Dr. Juan Manuel Muñoz-Céspedes. Unidad de Daño Cerebral. Hospital Beata María Ana. Vaquerías, 7. E-28011 Madrid. E-mail: jmcespedes@correo.cop.es

(C) 2004, REVISTA DE NEUROLOGÍA cación de las alteraciones encontradas. Autores como Holland [4] o Hagan [5] comenzaron a relacionar estas alteraciones con déficit cognitivos; más concretamente, planteaban que la pérdida de habilidad comunicativa era una consecuencia secundaria de problemas de índole cognitivo.

Esta idea marcó un hito para el desarrollo de investigaciones en el campo de la Neurolingüística y la Neuropsicología durante los noventa del siglo xx y principios de sigloxxi. Fundamentalmente, las investigaciones se han centrado en varias áreas de interés:

1. Definir y concretar el objeto de estudio, las HP y discursivas.

2. Identificar las estructuras corticales y subcorticales y los circuitos que subyacen a estas habilidades.

3. Determinar la influencia que ejercen sobre ellas otro tipo de funciones cognitivas, como la memoria, la atención y las FE.

4. Describir las alteraciones observadas en personas con TCE y las implicaciones que éstas tienen en su integración sociolaboral.

5. Desarrollar protocolos apropiados de evaluación.

6. En los últimos años, elaborar programas de tratamiento dirigidos a rehabilitar o compensar la pérdida de estas habilidades.

La participación de diferentes disciplinas en el desarrollo de la pragmática ha dado lugar a una ingente cantidad de términos que han provocado gran confusión entre los investigadores [6,7]. La pragmática es el uso del lenguaje, oral y gestual, en un determinado contexto. Snow y Ponsford [6] definen la competencia pragmática como el conjunto tanto de habilidades conversacionales como de habilidades interpersonales. Por otra parte, Penn [7] considera que la competencia pragmática la constituyen tres componentes:

1. Tres clases de conocimientos que son prerrequisitos para poder realizar una comunicación adaptada adecuadamente al contexto: conocimiento del lenguaje y su estructura, conocimiento del mundo y de los objetos y acciones dentro de 
ese mundo, y el conocimiento social o de las reglas que gobiernan la conversación y la conducta de un hablante. Considera que estos conocimientos conforman un sistema dinámico de partes inseparables e interactivas.

2. La adaptación: sucede cuando un organismo se sitúa en un contexto. El grado y éxito de tal adaptación depende de la integridad del organismo y de la naturaleza del contexto. Este componente se evalúa a partir del tiempo empleado en adaptarse, el mecanismo de adaptación empleado y el estado final tras la adaptación.

3. El procesamiento de variables: la comunicación es una conducta que se desarrolla en tiempo real y precisa ajustes constantes a partir del procesamiento constante que hace del contexto. Requiere un sistema muy preciso que sea capaz de desplegar de forma simultánea recursos como la atención, la retroalimentación y las FE.

Por tanto, la competencia pragmática incluye un conjunto de habilidades interrelacionadas que se manifiestan en tiempo real en forma de conductas adaptativas y que son controladas por unos procesos cognitivos subyacentes [7].

Por otra parte, los avances en las técnicas de análisis del discurso y su aplicación en la población afectada con daño cerebral han permitido identificar, en los últimos 15 años, diversas alteraciones en el uso que hacen estas personas del lenguaje dentro del contexto comunicativo. Más concretamente, la pérdida de HP subsiguientes a un TCE se manifiesta en un discurso caracterizado por: un lenguaje excesivamente prolijo, dificultades para promover, mantener o cambiar adecuadamente el tema de conversación, comentarios irrelevantes e indiscretos, duración excesiva del turno de habla, reducida sensibilidad a los indicios contextuales o verbales, dificultades en la adaptación del tono del habla y el vocabulario al contexto, problemas con la comprensión del lenguaje abstracto (metáforas, analogías, sarcasmo, etc.), dificultad para ordenar el discurso de forma secuencial y lógica, expresiones faciales y gestuales inadecuadas, problemas para manejar el espacio interpersonal, etc. [1,6-15]. Por tanto, pueden encontrarse dificultades en la modalidad expresiva y en la comprensiva. El perfil de alteraciones varía tanto en la modalidad como en el grado de afectación; es decir, pude haber alteraciones en el nivel expresivo pero no en el comprensivo, o al contrario, y pueden aparecer diferentes grados de afectación dentro de cada modalidad [16].

En relación con el estudio de las HP y su alteración tras un daño cerebral, ha existido en los últimos años un manifiesto y creciente interés por determinar la implicación que tiene en ellas el hemisferio derecho. En este sentido, se ha investigado el discurso de las personas con daño cerebral en el hemisferio derecho y se describe como tangencial, no informativo, irrelevante, repetitivo, confabulatorio o intrusivo, copioso o excesivamente recargado, inapropiado y disgregado. Igualmente, se han observado dificultades en la comprensión de la idea principal de las historias y las fábulas, problemas para manejar el significado, en la detección y comprensión del lenguaje metafórico, del sarcasmo, de las metáforas y las bromas, y para aprovechar la información afectiva que se transmite. Penn [7] considera que la coexistencia de déficit no lingüísticos, como la negligencia, las alteraciones atencionales o los déficit visuoespaciales, podría explicar, en cierta medida, la presencia de alteraciones pragmáticas en estos pacientes.

Continuando la línea abierta por Holland [4] y Hagan [5], autores como Hartley [17] -quien empleó por primera vez el término de 'alteraciones cognitivas-comunicativas'-McDonald
[10,11], Coelho et al [18] y otros han investigado las relaciones existentes entre los déficit cognitivos y las alteraciones discursivas. Se sabe que las alteraciones en la memoria, en la atención y en las FE influyen en las habilidades comunicativas.

Respecto a la memoria, aunque las investigaciones son muy escasas, se ha encontrado que existe una relación entre la memoria de trabajo y la comprensión [19-21]. En estos últimos trabajos, a pesar de que no había diferencias en la comprensión de dos tipos de discursos diferentes entre los tres grupos estudiados (lesiones en el hemisferio derecho, en el izquierdo y controles), sí se encontró una correlación significativa entre la capacidad de la memoria de trabajo y la comprensión global del discurso. Más concretamente, parece que la memoria de trabajo se asocia con la capacidad de los pacientes con TCE para generar inferencias a partir de historias [21].

En cuanto a la atención, se ha observado que sus limitaciones se relacionan con la comprensión del lenguaje figurativo y, especialmente, a las peticiones indirectas no convencionales [22], y con el manejo del lenguaje expresivo y receptivo en los pacientes con TCE [23].

Sin embargo, la mayoría de las investigaciones en este campo se han ocupado de la relación entre las FE y las alteraciones pragmáticas. Así, Ylvisaker y Szekeres [24] observaron que los sujetos que habían sufrido un TCE presentaban alteraciones discursivas atribuibles a problemas de carácter ejecutivo. Por ello, elaboraron una lista de aspectos de las FE cuya alteración podría afectar a la producción discursiva de los pacientes con TCE. Esta lista incluía: establecimiento de objetivos y autoconciencia, planificación, dirección e iniciativa, inhibición, monitorización, evaluación y flexibilidad en la solución de problemas. Por otro lado, las dificultades para monitorizar y planificar su producción verbal, afectan a la habilidad que tienen las personas con TCE para elaborar un discurso narrativo y procedimental [6]. Coelho et al [12] encontraron correlaciones significativas entre la estructura de la historia narrada y las FE; es decir, las personas con TCE mostraban dificultades para elaborar la secuencia de acontecimientos de una historia. Sin embargo, no observaron relaciones entre la cohesión y la producción de enunciados con las FE. Según estos autores, esto podría explicarse porque son elementos de naturaleza diferente. La cohesión y la producción de enunciados tiene una base más linguiística y la estructura más cognitiva. McDonald [10], en un estudio de casos del discurso procedimental de dos sujetos con TCE, encontró que ambos presentaban problemas para dar una explicación organizada, clara y efectiva a sus interlocutores. Sus ejecuciones discursivas ponían de manifiesto las características observadas en sus perfiles de FE alteradas. Otros autores $[11,25]$ han encontrado que los déficit en la inhibición producen alteraciones en el tipo de discurso que desarrollan los pacientes con TCE. Así, cuando los pacientes tenían que emplear peticiones indirectas no podían evitar ser más explícitos y contar su verdadero motivo [11] o tenían dificultades para elaborar peticiones que fueran novedosas teniendo en cuenta la posible resistencia del oyente a acceder a ellas; tendían a dar detalles que aumentaban la resistencia del interlocutor [25]. También se ha puesto de relieve cómo la pérdida de las habilidades de abstracción va a influir en la capacidad para interpretar y utilizar las inferencias conversacionales [26]. Por ello, les cuesta comprender los actos de habla indirectos, tanto convencionales como no convencionales, aunque estos últimos son más complejos para ellos [11,27]. 


\section{EVALUACIÓN DE LAS HABILIDADES PRAGMÁTICAS}

La evaluación de las HP de los pacientes con TCE se engloba dentro de la evaluación neurolingüística, la cual debe comenzar con una historia clínica del sujeto donde se registren los datos de filiación del paciente y de la lesión, el historial médico previo, el nivel educativo alcanzado y la ocupación. Junto a estos datos, conviene incluir y tener en cuenta otras alteraciones en tres dimensiones: la física y sensorial, la cognitiva y la emocional y motivacional, como señalan Muñoz-Céspedes et al [28], ya que afectan a la evaluación y a la interpretación posterior de los resultados de la misma. También es importante en este sentido poder recoger la mayor información sociocultural (su bagaje personal, social, educacional, cultural, de ocio y vocacional) que sea posible, que nos permita entender mejor y de una forma más global los resultados obtenidos y las implicaciones de su enfermedad [29,30].

Aunque se sabe que los pacientes con TCE presentan problemas para recuperar etiquetas léxicas, limitaciones en la fluidez verbal, parafasias y alteraciones en la comprensión verbal, etc., el empleo de las baterías tradicionales encubre las dificultades que experimentan estos pacientes para utilizar el lenguaje en contextos interactivos y comunicativos, ya que estas pruebas miden habilidades lingüísticas de forma aislada, y raramente aparecen como tal en el contexto comunicativo de la vida diaria.

Como en toda evaluación del lenguaje, se recomienda el empleo de grabaciones, bien en cinta o bien en vídeo, porque permiten el análisis detallado y preciso de las muestras, sirven de modelo inicial con el que comparar los avances del paciente y pueden emplearse además como material de trabajo en las sesiones de rehabilitación. Huelga decir que para llevar a cabo esto se necesita el consentimiento por escrito del paciente o de su tutor legal.

La variedad de disciplinas que se han interesado en el estudio de la competencia pragmática en los pacientes con TCE ha dado lugar a una amplia gama de pruebas y protocolos para su evaluación. Penn [7] establece varias dimensiones a lo largo de las cuales se pueden situar todas estas pruebas. Por tanto, como aparece en la figura 1, se pueden clasificar sobre la base de:

- La existencia o no de un modelo teórico subyacente.

- La estandarización o no de la misma.

- La medida de una o de varias habilidades.

- El análisis del resultado o del proceso.

A partir de la clasificación internacional del funcionamiento y la discapacidad (CIDDM-2) de la Organización Mundial de la Salud [31] es posible mostrar de forma didáctica tres formas de evaluar la actividad lingüística. Según la CIDDM-2, la salud y los estados relacionados con ésta pueden describirse a partir de dos componentes: funcionamiento y discapacidad y factores contextuales. Dentro del primer componente se encuentran tres partes: funciones y estructuras corporales (funciones fisiológicas y psicológicas y estructuras anatómicas), actividades (ejecución de una tarea o acción por una persona) y participación (acto de involucrarse en una situación vital). Los aspectos negativos de éstas según la clasificación serían, respectivamente: deficiencias o impairment (problemas en las funciones o en las estructuras corporales), limitaciones en la actividad o disability en la antigua clasificación (dificultades que un individuo puede tener en el desempeño/realización de actividades), y restricciones en la participación o handicap en la antigua clasificación

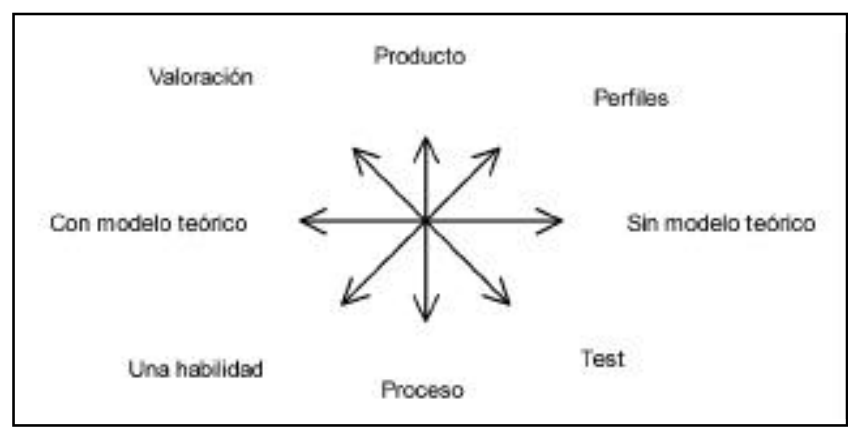

Figura. Dimensiones a lo largo de las cuales varían los protocolos de evaluación pragmática.

(problemas que un individuo puede experimentar al involucrarse en situaciones vitales).

Por tanto, las baterías tradicionales se ocupan de la valoración de la deficiencia. Son pruebas donde se mide la ejecución de determinadas habilidades linguísticas aisladas, como la denominación, la repetición, etc., y que van a detectar diferentes problemas de lenguaje que tienen las personas con daño cerebral. Pero, como se señalaba anteriormente, llevar a cabo una valoración de la competencia comunicativa de los pacientes con TCE basándose en los resultados obtenidos en estas pruebas puede dar lugar a falsos positivos, ya que no son instrumentos de medida del uso que hacen del lenguaje dentro de la interacción comunicativa.

Siguiendo esta argumentación, la falta de sensibilidad de dichas baterías impulsa el desarrollo de nuevas pruebas que reflejen cómo se ve afectado el uso del lenguaje en diferentes situaciones; es decir, que midan las limitaciones en la actividad comunicativa de los TCE. Dentro de esta dimensión se encuentran pruebas como el pragmatic protocol de Prutting y Kirchner [32] o el clinical discourse analysis de Damico [33]. No obstante, estas pruebas, aun cuando aportan ventajas importantes, no están exentas de limitaciones, entre las que cabe destacar que se evidencia todavía la artificialidad en la medida, ya que ésta se toma en el despacho, donde la interacción es semiestructurada, las demandas cognitivas son diferentes a las que tienen lugar en el contexto comunicativo real (procesamiento paralelo, indicios explícitos o implícitos que permiten regular la conducta verbal y gestual, etc.), existe una eliminación de distractores auditivos y visuales, y hace imposible la posibilidad de percibir su actuación en función de los papeles que desempeña en la vida (como hermano, hijo, marido u otros), etc. [6]

La atención se centra entonces en las restricciones de la participación, en buscar una visión más integradora y holística de la habilidad comunicativa del paciente con TCE y en determinar las necesidades y demandas comunicativas en los diferentes contextos en los que se desarrolla su vida. Para llevar a cabo la evaluación de estos aspectos, se propone emplear técnicas de análisis etnográficas, como la observación participante -donde el clínico acompaña al paciente dentro de un contexto que para él es relevante y observa las dificultades y los aspectos positivos que aparecen dentro de este contexto- o la triangulación -donde se emplean métodos cualitativos para el registro de los datos, como formularios o protocolos cumplimentados por diferentes informadores que, a partir del análisis conjunto, ofrecen los aspectos deficitarios para el paciente-, estudios fenomenológicos, narraciones, etc. Body y Parker [34] señalan que, si bien esta evaluación de campo tiene un valor significativo para la comprensión 
Tabla I. Pruebas empleadas en la evaluación del uso del lenguaje en contextos naturales.

Evaluación pragmática

Pragmatic protocol (Prutting y Kirchner, 1987) [35]

Discourse abilities profile (Terrell y Ripich, 1989) [36]

Edinburgh functional communication profile (Skinner et al 1984) [37]

Communicative abilities in daily living (Holland, 1980) [38]

Conversation analysis profile for people with

cognitive impairments (Perkins et al 1997) [39]

Evaluación funcional

Sarno's functional communication profile (Samo, 1975) [40]

The rating of functional performance (Wertz et al, 1981) [41]

The communicative competence evaluation instrument (Houghton et al 1982) [42]

The Amsterdam-Nijmegen everyday language test (Bloment, 1990) [43]

The functional linguistic communication inventory

(Bayles y Tomoeda, 1995) [44]

Functional assessment of communication skills

for adults (Frattali et al, 1995) [45]

Communicative effectiveness index (Lomas et al, 1989) [46]

Questionnaire rating personal/communicative style

(Swindell et al, 1982) [47]

del caso ante el que nos encontramos, en la práctica clínica es muy complicada de llevar a cabo. Por ello, plantean recurrir a múltiples informadores, que incluyen, por supuesto, a familiares, cuidadores, personas próximas al paciente e incluso la información que pueden aportar el resto de los miembros del equipo multiprofesional. Con ello, se pretenden registrar interacciones comunicativas diversas, donde se pongan de manifiesto los diferentes papeles, géneros discursivos, características comunicativas, adaptaciones, etc., y mitigar los posibles 'sesgos' que pueden aparecer durante la evaluación por parte del clínico.

En una línea de argumentación parecida, se establece una división entre lo que sería la 'evaluación pragmática' y la 'evaluación funcional'. Respecto de la primera, hay que indicar que se basa en modelos teóricos multidimensionales, que incluyen elementos lingüísticos y no lingüísticos y que exploran este intervalo de conductas para explicar la variación comunicativa en diferentes contextos. Mientras que respecto a la segunda, es imprescindible señalar que se trata de pruebas en las que se mide el efecto o el impacto de las alteraciones pragmáticas en el ámbito social y vocacional o se requiere la estimación de la eficiencia comunicativa por parte de familiares o personas cercanas al paciente. En la tabla I se presentan, por separado, las pruebas que se incluirían en cada una de las formas de evaluación [35-47].

Como se observa en la tabla, entre las pruebas más conocidas de evaluación pragmática se encuentra el análisis del discurso. Coelho [48] resume detalladamente qué aspectos pueden analizarse a partir de la muestra de habla de cinco o más frases grabadas en cinta o en vídeo y transcritas en cláusulas independientes, con alguna cláusula dependiente de éstas. El análisis puede ser microlingüístico o macrolingüístico.
En cuanto a los elementos del análisis microlingüístico, se incluyen los aspectos fonológicos (precisión articulatoria, errores fonológicos, etc.), de producción léxica (problemas de evocación espontánea, parafasias, etc.) y sintáctica (número de oraciones simples, número de oraciones subordinadas, número de subordinadas entre oraciones simples en diferentes muestras, competencia gramatical de frase: frecuencia de oraciones agramaticales por muestra, etc.).

En el análisis macrolingüístico se miden la cohesión, la estructuración de las historias y la coherencia. El estudio de la cohesión comprende tres pasos:

1. Identificar la palabra que se usa como nexo cohesivo.

2. Clasificar el nexo dentro de las categorías gramaticales (referencia: personal, demostrativo o comparativo; conjunción: causales, adversativas, temporales o copulativas; léxica: reiteración; elipsis, y sustitución). Cada género discursivo (conversacional, narrativo y procedimental) requiere diferentes patrones de cohesión, por lo que una comparación entre ellos aportará más datos interesantes.

3. Determinar la adecuación de la función del nexo en un texto dado (completo: cuando el referente es fácil de encontrar y no plantea ambigüedad; incompleto: cuando el referente no está en el texto; erróneo: cuando se produce ambigüedad) [48].

Por último, se han considerado una serie de cuestiones metodológicas muy interesantes que deberían tenerse en cuenta durante el proceso de evaluación de las HP. Por ejemplo, la evaluación de éstas en términos cuantitativos del tipo 'apropiado' o 'inapropiado' lleva implícita la toma de un modelo de comunicación normal subyacente, que en principio todavía no se ha establecido claramente, a partir del cual, y en función de la desviación del mismo se establecen criterios cuantitativos de alteraciones. El modelo de normalidad se basa en el concepto de tendencia central y los clínicos perciben desviación cuando las conductas observadas son discrepantes con su modelo de tendencia central. Uno de los problemas que se plantean en este sentido es: ¿en qué momento decide un clínico que determinado comportamiento es discrepante del modelo normal? Más aún cuando dicho modelo no se ha definido bien. Para abordar este problema se plantea como alternativa [49] llevar a cabo una evaluación donde se tengan en cuenta los papeles que adopta el paciente, el contexto en el cual se desarrolla, el tipo de relación comunicativa que se establece entre el clínico y el paciente, su bagaje social, nuestros estigmas y estereotipos al interpretar las conductas y la existencia de diferencias individuales.

\section{CUESTIONES GENERALES EN LA REHABILITACIÓN DE LAS HABILIDADES PRAGMÁTICAS}

La recuperación de un paciente con TCE comienza ya en los primeros momentos tras la lesión. A lo largo de los meses subsiguientes se produce el mayor grado de recuperación funcional, que en pocas ocasiones llega a ser completo -sobre todo en los TCE graves-. Por tanto, tras este período de recuperación espontánea persiste una serie de déficit que van a precisar una rehabilitación de distinta índole, según el caso, y que sería conveniente comenzar lo antes posible.

Como es bien sabido, existen tres abordajes de rehabilitación cognitiva que se diferencian en cuanto a su finalidad. Se pueden resumir como sigue: 
- Restablecimiento: busca la restauración de la función perdida a través del reaprendizaje de la misma.

- Sustitución: como su nombre indica, pretende la realización de una función mediante aquellas habilidades cognitivas que se preservan, en la medida de lo posible.

- Compensación: intenta dotar a la persona de aquellas funciones perdidas mediante adaptaciones o ayudas externas.

En principio, la elección de una u otra forma de rehabilitación dependerá de las características de cada caso concreto, de la gravedad de la lesión, del tiempo transcurrido desde el accidente, etc. [50]. El objetivo de la rehabilitación debería ser 'reducir las limitaciones en la actividad e incrementar la participación', considerando que la rehabilitación de los déficit cognitivos de forma aislada no supone una mejora directa en contextos reales, donde entran en juego las dificultades de generalización de estos pacientes y el aumento de demandas cognitivas por parte de los contextos. Por ello, se propone una rehabilitación más global, donde, desde un primer momento se busque la recuperación y la adaptación funcional y se evite que los pacientes lleguen a considerar los procedimientos compensatorios como última alternativa terapéutica, con los efectos negativos que esto tiene sobre las expectativas de éxito y la motivación del paciente hacia la rehabilitación.

El programa de rehabilitación para las HP debería incluir tanto sesiones individuales como sesiones en grupo. Ambas formas ofrecen la posibilidad de abordar aspectos diferentes pero complementarios entre sí. Las sesiones individuales permiten trabajar los aspectos relevantes para el paciente concreto, establecer una relación satisfactoria entre el terapeuta y el paciente, manifestar las dificultades encontradas y las preocupaciones personales, plantear los objetivos de forma conjunta, etc. Mientras, en las sesiones de grupo se abre la posibilidad de poner en práctica aquellas habilidades aprendidas, manejar una cantidad mayor de demandas cognitivas y comunicativas que en las sesiones individuales, les preparan para ser capaces en un contexto real de adaptar su comunicación a diferentes interlocutores y a diferentes situaciones, aprenden de otros pacientes, etc. La distribución de las sesiones no tiene por qué ser secuencial (primero individuales y posteriormente grupales) sino que pueden simultanearse para reforzar y adaptar o modificar los aprendizajes alcanzados en cada una de ellas [6,29]. Una de las características principales de estos programas es el trabajo explícito e implícito de las habilidades metalinguiísticas y metacognitivas relacionadas con la comunicación que se presupone que se alteran en los pacientes con daño cerebral y que favorecen un manejo más adecuado de las capacidades y habilidades preservadas durante la comunicación [7].

Ya en la evaluación se resaltó la presencia de dificultades de otra índole (físicas, neuropsicológicas, etc.), que iban a condicionar tanto el proceso de evaluación como la interpretación de los resultados obtenidos en la misma. Esto es así porque el individuo es más que la suma de un conjunto de características; la persona debe entenderse como un todo, como un ente global. De ahí que, también desde la rehabilitación. se deba llevar a cabo un abordaje conjunto de los problemas que presenta, en el cual participen aquellas disciplinas que puedan ser significativas para la mejora de la calidad de vida de los pacientes con TCE. Por tanto, debe desarrollarse una comunicación fluida entre los profesionales que atienden a estas personas a partir del estudio conjunto y detallado de cada caso. Los distintos miembros del equipo no sólo aportan información relevante, sino que también participan en el propio proceso de rehabilitación, ya que son interlocutores potenciales para el paciente y promueven contextos sociales donde están implicados aspectos lingüísticos, como por ejemplo tareas de rehabilitación centradas en actividades de la vida diaria, como hacer la compra, acudir al médico, solicitar información sobre horarios de autobuses, etc.

Así, la logopedia debe estar abierta a las aportaciones del resto de los profesionales, a las posibilidades que ofrece el trabajo en equipo para desarrollar y practicar intercambios comunicativos, y al trabajo tanto con el paciente como con la familia. En este sentido, debe establecerse una comunicación relajada, abierta, respetuosa y fluida con los familiares y/o cuidadores de los pacientes, donde se puedan tratar aspectos relacionados con las limitaciones del paciente, las implicaciones de éstas en la comunicación, el desarrollo de ambientes favorecedores de la fluidez comunicativa, el establecimiento conjunto de aspectos prioritarios para la rehabilitación de forma coordinada con el paciente -si esto es posible-, etc. Esta comunicación debe ser bidireccional, ya que es igual de importante dar información que permita entender y manejar las dificultades del paciente como conocer las limitaciones que en un contexto clínico pueden no evidenciarse, y las que surgen a lo largo del proceso de intervención y que en un principio pueden no presentarse.

En las sesiones de rehabilitación, el logopeda y el paciente deben establecer una relación empática y basada en un respeto mutuo, para poder afrontar aspectos relacionados con los déficit del paciente, con el establecimiento conjunto de los objetivos de la rehabilitación, la aceptación de las posibles secuelas, etc. Se recomienda la implicación del paciente en el proceso de control y ajuste de los objetivos y de los avances, ya que permite que perciba las metas como parte suya y se motive para alcanzarlas. Para llevar a cabo tal implicación, Snow y Douglas [29] proponen establecer conjuntamente los objetivos concretos para cada sesión al inicio de la misma, para poder registrar al final de ésta los progresos realizados, de forma que el paciente pueda constatar gráfica y vivencialmente los avances y las dificultades. Este procedimiento de evaluación puede aplicarse dentro de una sesión y a lo largo del proceso de intervención. Sin embargo, hay que tener en cuenta que en los momentos iniciales de la rehabilitación el clínico puede encontrar que las expectativas del paciente no son muy realistas, que algunos pacientes no consiguen a lo largo de su rehabilitación alcanzar un nivel apropiado de conciencia de sus limitaciones o que la relación esfuerzo/beneficio lleve al paciente a abandonar el proceso rehabilitador. Por ello, resulta imprescindible tener en cuenta estos factores en el momento de establecer los objetivos conjuntamente.

Finalmente, hay que mencionar que la efectividad de los programas de rehabilitación de HP es un tema muy complejo, por varias cuestiones:

- Por la dificultad para aplicar programas de rehabilitación estandarizados, dada la diversidad de necesidades y contextos en que se llevan a cabo las intervenciones, lo que dificulta el establecimiento de comparaciones intersujetos.

- Por la diversidad de los instrumentos de medida empleados y la falta de sensibilidad de algunas pruebas para detectar los cambios experimentados por los pacientes.

- Porque la necesidad de incluir a varios informadores hace que la percepción de los logros alcanzados varíe significativamente; dicho de otro modo, la valoración del grado de recuperación no siempre coincide entre los clínicos, el paciente y los allegados [29]. 
Tabla II. Consideraciones ante un discurso narrativo o procedimental (adaptado de Swnow y Douglas [29]).

¿Cuánto sabe o conoce este oyente del tema de la conversación?

¿De cuánto tiempo dispongo para expresar mis ideas?

Si he usado un pronombre (p. ej., 'él, nosotros,ellos'), ¿he dicho primero a quién me refiero?

¿Comencé desde el principio y pasé de una idea a la siguiente de un modo lógico?

¿Permanezco dentro del tema o me voy por las ramas?

¿Repito alguna información innecesariamente?

¿Incluí todos los pasos y/o elementos que quería trasmitir?

¿Qué tipo de señales no verbales ofrece el oyente sobre su grado

de interés en el tema? (considere el contacto visual, la agitación o movimientos, los intentos de escape y/o evitación)

¿Parece el oyente seguir lo que digo? (Observe los signos de entendimiento realizados con la cabeza o los signos de confusión que indiquen que el oyente 'se ha perdido')

¿Pregunté al oyente si tenía alguna pregunta

o si le gustaría clarificar alguna cuestión?

\section{PRINCIPALES APROXIMACIONES TERAPÉUTICAS}

Dentro de las diferentes aproximaciones a la rehabilitación de las $\mathrm{HP}$, existe un grupo de programas de rehabilitación que se centran en aspectos relacionados con la estructura verbal del discurso y con los elementos paralingüísticos que lo acompañan. En ellos, se propone hacer explícitos los componentes de los diferentes tipos de discurso (narrativo, procedimental y conversacional) y sus finalidades para discutirlos y trabajarlos junto con el paciente. Así, se plantea una segmentación de los elementos que conforman los discursos para poder manejarlos didácticamente en las sesiones de rehabilitación, donde se trabajan en los primeros momentos dentro de una estructura definida, para pasar después a introducir variaciones con el fin de abarcar las diferentes variedades discursivas que aparecen en la vida real. Las sesiones de rehabilitación pueden ser individuales o grupales y en ellas se emplean técnicas de modelado, role play, autoanálisis, retroalimentación por parte de otros pacientes participantes en la terapia o del terapeuta, visionado de vídeos, prácticas en contextos reales, etc. Otros instrumentos terapéuticos que se proponen son ayudas externas que se retiran progresivamente, como, por ejemplo, tarjetas donde aparezcan frases cortas que ayuden a resolver problemas como el discurso prolijo (p. ej., 'sé breve'), la impulsividad y desorganización (p. ej., 'para y piensa'), etc. [6,29], tal y como sucede también en los programas de rehabilitación de las FE.

Stein y Glenn [51] proponen, para rehabilitar el discurso narrativo, que se le enseñe al paciente a identificar los diferentes elementos que constituyen una narración, con el empleo de sistemas gráficos de marcación que le permitan, en primer lugar, estructurar mentalmente su discurso y, en segundo lugar, comprender y utilizar las relaciones lógicas, causales, secuenciales, etc., que se dan en las narraciones, así como conocer cuáles son los elementos gramaticales que permiten establecer tales relaciones y aplicarlos adecuadamente.

Otros autores [29] resumen las cuestiones más relevantes que un paciente debe tener en cuenta a la hora de desarrollar tanto un discurso narrativo como un discurso procedimental. Estos elementos aparecen resumidos en la tabla II.
Entre las aportaciones terapéuticas más empleadas conviene citar el denominado programa de entrenamiento de la competencia social, que no se centra sólo en las habilidades comunicativas, sino que también incluye el trabajo sobre los 'principios comunicativos'. Según los autores, estos principios son universales, constantes y existen de forma independiente a las 'habilidades comunicativas', entendidas éstas como conductas comunicativas que se manifiestan en diferentes contextos sociales a través de un estilo de interacción adaptado a ellos. La conjunción de ambos elementos da lugar a la 'flexibilidad comunicativa', es decir, la capacidad tanto de reconocer la necesidad de ajustar las conductas comunicativas en función al contexto como de llevar a cabo dichos ajustes.

Esta aproximación se basa en el principio de que una mayor comprensión de la comunicación y del papel que ésta desempeña en el desarrollo y mantenimiento de relaciones productivas, independientemente del contexto en que tengan lugar, permitirá que los pacientes alcancen la flexibilidad comunicativa suficiente para ser capaces de establecer y sostener relaciones comunicativas a través de gran variedad de contextos.

El programa se desarrolla en cinco fases diferentes, que se presentan en la tabla III. En principio, tienen un orden secuencial, aunque puede modificarse en función de las necesidades de la rehabilitación. Principalmente, se orienta a grupos de pacientes ambulatorios con un déficit neuropsicológico moderado, coordinados por uno o dos 'facilitadores'. Éstos participan en las sesiones estableciendo una relación comunicativa simétrica con los pacientes, en la que, por una parte, coordinan las discusiones y las actividades, ayudan a promover el autoexamen y la capacidad de los pacientes para proporcionarse retroalimentación de forma constructiva, etc., y, por otra, comparte sus conocimientos sobre la comunicación y sobre su propia forma de comunicarse y acepta la retroalimentación relacionada con su conducta comunicativa ofrecida por los pacientes [52].

Los programas dirigidos a compensar las dificultades comunicativas de los pacientes con TCE son muy heterogéneos, ya que el perfil de limitaciones del paciente y los contextos comunicativos en los cuales se va a desenvolver va a determinar el tipo de ayudas compensatorias que precise. Turkstra y Flora [53] describen un programa de rehabilitación elaborado para un paciente con TCE que presentaba alteraciones en las FE; más concretamente, tenía dificultades para organizar información, junto con alteraciones en la atención dividida, en flexibilidad cognitiva, dificultades para diferenciar la información relevante de la irrelevante y para resolver conflictos de planificación. El paciente desarrollaba su actividad laboral como trabajador social, pero estas dificultades influían significativamente en la manera en que realizaba las entrevistas y en la redacción de los informes, lo cual le impedía mantener su trabajo. El programa se desarrolló en 21 sesiones de una hora a lo largo de 10 semanas, donde, a través de role play, se pusieron en práctica los protocolos elaborados para desarrollar las entrevistas, para registrar la información y para elaborar el informe. En ellos se reflejaron los datos relevantes que deben recogerse en las entrevistas realizadas por trabajadores sociales. Las sesiones incluían conversación espontánea (cinco minutos), escritura al dictado (cinco minutos) y elaboración de un informe escrito (50 minutos) sobre diferentes casos tomados del DSM-IV. Tras la rehabilitación, se encontró que las mejoras en las FE redundaron en una mayor habilidad comunicativa y en una elaboración de los infor- 
mes más apropiada, que le sirvieron para obtener y mantener un nuevo empleo como trabajador social.

\section{CONCLUSIONES}

Las consecuencias sociales y laborales a largo plazo de las dificultades comunicativas de las personas que han sufrido un TCE, junto con las limitaciones de los protocolos de evaluación tradicionales, ha llevado en las últimas décadas hacia un interés creciente en la elaboración de instrumentos de medida más adecuados para detectar las alteraciones pragmáticas y el diseño de programas de rehabilitación que ayuden a los pacientes a minimizar y compensar estos déficit.

Sin embargo, todavía existen muchos aspectos que precisan un mayor desarrollo para ofrecer una mejor comprensión de las dificultades ante las que nos encontramos, como es el caso de la interacción con otras habilidades cognitivas (de memoria, atención y FE) y la comunicación en situaciones sociales de mayor complejidad. Por otra parte, se evidencia la escasez tanto de instrumentos de evaluación como de programas de investigación de estos problemas para población de habla española.

Por último, se señala que la intervención de estas dificultades no puede afrontarse desde un único profesional, sino que deben implicarse todos los miembros del equipo y deben elaborarse programas integrados, en los que se incluyan elementos relacionados con conocimientos generales sobre el lenguaje, el mundo y la comunicación, con las capacidades de flexibilización y adaptación de la conducta a diferentes situaciones y con el control y tratamiento de los recursos cognitivos durante la comunicación.
Tabla III. Fases del programa de entrenamiento de la competencia social.

Fases Contenidos

Fase 1: discusión grupal: Se discuten los principios de la comunicación ¿qué es comunicación? para tomar conciencia de que son universales y de la repercusión de los estilos comunicativos de cada paciente en su propia vida y en sus relaciones sociales

Fase 2: rellenar un cuestionario sobre habilidades de

Se pide a los pacientes que rellenen en grupo comunicación social un cuestionario sobre sus habilidades y sus dificultades y se les anima a darse retroalimentación mutua de forma constructiva

Fase 3: elaborar los objetivos comunicativos

Se le pide a cada paciente que identifique tres objetivos de rehabilitación que le qustaría trabajar. El terapeuta ayuda en la elección y establece también tres objetivos para él. Éstos se registran por escrito en una escala de logros que se emplea para que el paciente monitorice sus progresos y anote datos de las sesiones. No debe memorizar los objetivos

\begin{tabular}{ll}
\hline $\begin{array}{l}\text { Fase 4: trabajar papeles } \\
\text { y actividades de } \\
\text { simulación }\end{array}$ & $\begin{array}{l}\text { Se diseñan actividades comunicativas donde } \\
\text { desempeñen sus objetivos. Las propuestas } \\
\text { de actividades parten de los pacientes (aquellas } \\
\text { a las que se enfrentan o se van a enfrentar) } \\
\text { y del propio profesional (hablar por teléfono, } \\
\text { pedir direcciones, etc.). Se graban en vídeo } \\
\text { y se analizan y discuten de forma grupal }\end{array}$ \\
\hline Fase 5: generalizar & $\begin{array}{l}\text { Se generalizan fuera del lugar de rehabilitación } \\
\text { los objetivos alcanzados y los principios } \\
\text { los objetivos }\end{array}$ \\
& $\begin{array}{l}\text { las variables se controlan con dificultad. } \\
\text { Los contextos dependerán de cada caso } \\
\text { (clase de pintura, piscina, etc.) }\end{array}$ \\
\hline
\end{tabular}

\section{BIBLIOGRAFÍA}

1. Bond F, Godfrey HPD. Conversation with traumatically brain-injured individuals: a controlled study of behavioral changes and their impact. Brain Inj 1997; 11: 319-29.

2. Ojeda del Pozo N, Ezquerra-Iribarren JA, Urruticoechea-Sarriegui I, Quemada-Ubis JI, Muñoz-Céspedes JM. Entrenamiento en habilidades sociales en pacientes con daño cerebral adquirido. Rev Neurol 2000; 30: 783-7.

3. Sarno MT. The nature of verbal impairment after closed head injury. J Nerv Ment Dis 1980; 168: 685-92.

4. Holland AL. When is aphasia? The problem of closed head injury. In Brookshire $\mathrm{RH}$, ed. Clinical aphasiology conference proceedings. Minneapolis: BRK Publishers; 1982. p. 255-76.

5. Hagan C. Language disorders in head trauma. In Holland A, ed. Language disorders in adults. San Diego: College Hill Press; 1984. p. 245-81.

6. Snow P, Ponsford J. Assessing and managing changes in communication and interpersonal skills following TBI. In Ponsford J, ed. Traumatic brain injury: rehabilitation for everyday adaptative living. Hove, UK: Lawrence Erlbaum Associates; 1995. p. 137-64.

7. Penn C. Pragmatic assessment and therapy for persons with brain damage: what have clinicians gleaned in two decades? Brain Lang 1999; 68: 535-52.

8. Mentis M, Prutting CA. Cohesion in the discourse of normal and head injured adults. J Speech Lang Hear Res 1987; 30: 88-98.

9. Hartley LL, Jensen P. Narrative and procedural discourse after closed head injury. Brain Inj 1991; 5: 202-15.

10. McDonald S. Pragmatic language skills after closed head injury: ability to meet the informational needs of the listener. Brain Lang 1993; 44: 28-48.

11. McDonald S, Van Sommers P. Pragmatic language skills after closed head injury: ability to negotiate requests. Cogn Neuropsychol 1993; 10: 297-315.

12. Coelho CA, Liles BZ, Duffy RJ. Analysis of conversational discourse in head-injured adults. J Head Trauma Rehabil 1991; 6: 92-8.

13. Coelho CA. Discourse production deficits following traumatic brain injury: a critical review of the recent literature. Aphasiology 1995; 9: 409-30.

14. Prigatano GP, Roueche JR, Fordyce DJ. Neuropsychological rehabilita tion after brain injury. Baltimore: Johns Hopkins University Press; 1986.
15. Martinelli Gispert-Saúch M. Trastornos de la comunicación en el traumatismo craneoencefálico. Mapfre Medicina 2000; 11 (Supl 1): 130-4.

16. Turkstra L, McDonald S, Kaufmann P. Assessment of pragmatic communication skills in adolescents after brain injury. Brain Inj 1995; 10 . 329-45.

17. Hartley LL. Cognitive-Communicative abilities following brain injury: a functional approach. San Diego: Singular Publishing Group; 1995.

18. Coelho CA, Liles BZ, Duffy RJ. Impairment of discourse abilities and executive functions in traumatically brain-injured adults. Brain Inj 1995; 9: 471-7.

19. Van der Linder M, Poncelet M. Working memory and language. In Stemmer B, Whitaker HA, eds. Handbook of neurolinguistics. San Diego: Academic Press; 1998. p. 289-300.

20. Denis M, Barner MA. Knowing the meaning, getting the point, bridging the gap, and carrying the message: aspect of discourse following closed head injury in childhood and adolescence. Brain Lang 1990; 39: 428-46.

21. Tompkins CA, Bloise CGR, Timko ML, Baumgaertner A. Working memory and inference revision in brain-damaged and normally aging adults. J Speech Lang Hear Res 1994; 91: 185-215.

22. Stemmer B, Giroux F, Joanette Y. Production and evaluation of requests by right hemisphere brain-damage individuals. Brain Lang 1994; 7: 1-31.

23. Watt N, Penn C, Jones D. Speech-language evaluation of closed head injured subjects in South Africa: cultural applicability and ecological validity. S Afr J Commun Disord 1996; 43: 85-92.

24. Ylvisaker M, Szekeres SF. Metacognitive and executive impairments in head-injured children and adults. Topics in Language Disorders 1989; 9: $34-42$.

25. McDonald S, Pearce S. Requests that overcome listener reluctance: Impairment associated with executive dysfunction in brain injury. Brain Lang 1998; 61: 88-104.

26. Body R, Perkins M, McDonald S. Pragmatics, cognition, and communication in traumatic brain injury. In McDonald S, Togher L, Code C, eds. Communication disorders following traumatic brain injury. Hove, UK: Psychology Press; 1999. p. 95.

27. McDonald S. Differential pragmatic language loss after closed brain 
injury: ability to comprehend conversational implicature. Applied Psycolinguistic 1992; 13: 295-312.

28. Muñoz-Céspedes JM, Íñigo S, Melle N. Evaluación de la actividad lingüística y comunicativa después de un daño cerebral. In Bruna $\mathrm{O}$, Junqué C, Mataró M, eds. Neuropsicología del lenguaje. Barcelona: Masson [in press].

29. Snow P, Douglas J. Discourse rehabilitation following traumatic brain injury. In McDonald S, Togher L, Code C, eds. Communication disorders following traumatic brain injury. Hove, UK: Psychology Press; 1999. p. 271-320.

30. McGann W, Werven G. New management concept. Social competence and head injury: a new enphasis. Brain Inj 1995; 9: 93-102.

31. Organización Mundial de la Salud. Clasificación internacional del funcionamiento, de la discapacidad y de la salud (CIDDM-2). URL: http:// www3.who.int/icf/onlinebrowser/icf.cfm.

32. Pruttin CA, Kirchner D. A clinical appraisal of the pragmatic aspects of language. J Speech Hear Disord 1987; 52: 105-19.

33. Damico JS. Clinical discourse analysis: a functional approach to language assessment. In Simon CS, eds. Communication skills and classroom success. London: Taylor \& Francis; 1985.

34. Body R, Parker M. The use of multiple informants in the assessment of communication after traumatic brain injury. In McDonald S, Togher L, Code $\mathrm{C}$, eds. Communication disorders following traumatic brain injury. Hove, UK: Psychology Press; 1999. p. 147-74.

35. Prutting CA, Kirchner D. A clinical appraisal of the pragmatic aspects of language. J Speech Hear Disord 1987; 52: 105-19.

36. Terrell BY, Ripich DN. Discourse competence as a variable in intervention. Semin Speech Lang 1989: 10; 282-97.

37. Skinner C, Wirz S, Thompson I, Davidson J. Edinburgh functional communication profile. Scotland: Winslow Press; 1984.

38. Holland AL. Communicative abilities in daily living. Baltimore: Park Press; 1980.

39. Perkins L, Witworth A, Lesser R. Conversation analysis profile for people with cognitive impairments. London: Whurr; 1997.

40. Sarno MT. The functional communication profile. New York: NYU Medical Centre, Institute of Rehabilitation Medicine; 1975.

\section{ALTERACIONES DE LA PRAGMÁTICA DE LA COMUNICACIÓN DESPUÉS DE UN TRAUMATISMO CRANEOENCEFÁLICO}

Resumen. Objetivo. Presentar la contribución de las alteraciones cognitivas a la pérdida de la competencia comunicativa tras un traumatismo craneoencefálico (TCE), así como los instrumentos más empleados para evaluar las habilidades pragmáticas y las diferentes aproximaciones terapéuticas para reducir o compensar este tipo de déficit. Desarrollo. La revisión de la literatura ilustra de qué modo otros procesos cognitivos (atención, memoria y funciones ejecutivas) interactúan con las habilidades comunicativas, expresivas y comprensivas. Los avances en la evaluación de las personas con un TCE permiten identificar déficit tales como la presencia de un lenguaje excesivamente prolijo, con comentarios irrelevantes; dificultades para desarrollar una conversación; escasa sensibilidad a indicios contextuales o verbales; problemas para comprender el lenguaje abstracto (metáforas, analogías), etc. Se resumen los principales aspectos relacionados con su evaluación y tratamiento, y se destaca la necesidad de adaptar y desarrollar protocolos de exploración en nuestro idioma, así como emplear diferentes estrategias de rehabilitación basadas en el desarrollo de habilidades narrativas, el entrenamiento en principios de la comunicación, la mejora de la competencia social, etc., desde la perspectiva de la competencia pragmática. Conclusiones. La influencia que ejercen otros procesos cognitivos sobre la competencia comunicativa de estas personas y la importancia de estas habilidades en la integración social y laboral indican la conveniencia de aumentar nuestro conocimiento sobre los mencionados procesos de dichas interrelaciones. Se destaca la relevancia de los nuevos métodos de tratamiento, que permiten incrementar la calidad de vida de esta población y requieren la participación conjunta de distintos profesionales. [REV NEUROL 2004; 38: 852-9]

Palabras clave. Competencia lingüística. Evaluación neuropsicológica. Pragmática. Procesos cognitivos. Rehabilitación neuropsicológica. Traumatismos craneoencefálicos.
41. Wertz RT, Colling M, Weiss D, Kurtzke JF, Friden T, Brookshire RH, et al. Veterans administration cooperative study on aphasia: a comparison of individual and group treatment. J Speech Hear Disord 1981; 24: 580-94.

42. Houghton PM, Pettit JM, Towey MP. Measuring communicative competence in global aphasia. In Brooksihre RH, eds. Clinical aphasiology: conference proceedings. Minneapolis: BRK; 1982. p. 28-39.

43. Bloment $\mathrm{L}$. What functional assessment can contribute to setting goals for aphasia therapy. Aphasiology 1990: 4; 307-20.

44. Bayles K, Tomoeda C. Functional linguistic communication inventory. UK: Winslow Press; 1995.

45. Frattali CM, Thompson CM, Holland AL, Wohl BC, Ferketie MM. American speech-language-hearing association functional assessment of communication skills for adults. Rockville: ASHA; 1995.

46. Lomas J, Pickard L, Betser S, Elbard H, Finlaysond A, Zoghaib C. The communicative effectiveness index: development and psychometric evaluation of a functional communication measure for adult aphasia. J Speech Hear Disord 1989; 54: 113-224.

47. Swindell CS, Pashek GV, Holland AL. A questionnarie for surveying personal and communicative style. In Brooksihre RH, ed. Clinical aphasiology: conference proceedings. Minneapolis: BRK; 1982. p. 50-63.

48. Coelho CA. Discourse analysis in traumatic brain injury. In McDonald $\mathrm{S}$, Togher L, Code C, eds. Communication disorders following traumatic brain injury. Hove, UK: Psychology Press; 1999. p. 55-71.

49. McGann W, Werven G. New management concept. Social competence and head injury: a new emphasis. Brain Inj 1995; 9: 93-102.

50. Muñoz-Céspedes JM, Tirapu J. Rehabilitación neuropsicológica. Madrid: Síntesis; 2001.

51. Stein NL, Glenn CG. An analysis of story comprehension in elementary school children. In Feedle RO, ed. New directions in discourse processing. New Jersey: Ablex Publishing; 1979. p. 53-120.

52. McGann W, Werven G, Douglas M. Social competence and head injury: a practical approach. Brain Inj 1997; 11: 621-8.

53. Turkstra L, Flora TL. Compensating for executive function impairments after TBI: a single case study of functional intervention. J Commun Disord 2002; 35: 467-82.

\section{ALTERAÇÕES DA PRAGMÁTICA DA COMUNICAÇÃO APÓS TRAUMATISMO CRÂNIO-ENCEFÁLICO}

Resumo. Objectivo. Apresentar o contributo das alterações cognitivas à perda da competência comunicativa após o traumatismo crânio-encefálico (TCE), os instrumentos mais utilizados para avaliar as capacidades pragmáticas $e$ as diferentes abordagens terapêuticas para reduzir este tipo de défice. Desenvolvimento. A revisão da literatura ilustra de que modo outros processos cognitivos (atenção, memória e funções executivas) interagem com as capacidades comunicativas, expressivas e compreensivas. Os avanços na avaliação das pessoas com TCE permitem identificar défices tais como a presença de uma linguagem excessivamente prolixa, com comentários irrelevantes; dificuldades no desenvolvimento da conversação; fraca sensibilidade a indícios contextuais ou verbais; problemas na compreensão da linguagem abstracta (metáforas, analogias, etc.). Resumem-se os principais aspectos relacionados com a sua avaliação e tratamento, e destaca-se a necessidade de adaptar e desenvolver protocolos de exame no nosso idioma, assim como a utilização de diferentes estratégias de reabilitação baseadas no desenvolvimento de capacidades narrativas, treino em princípios da comunicação, melhoria da competência social, etc, sob a perspectiva da competência pragmática. Conclusões. A influência que exercem outros processos cognitivos sobre a competência comunicativa destas pessoas e a importância destas capacidades na integração social indicam a conveniência de aumentar o nosso conhecimento sobre os processos das referidas inter-relações. Destaca-se a relevância dos novos métodos de treino que permitem incrementar a qualidade de vida desta população e requerem a participação conjunta de profissionais distintos. [REV NEUROL 2004; 38: 852-9]

Palavras chave. Avaliação neuropsicológica. Competência linguística. Pragmática. Processos cognitivos. Reabilitação neuropsicológica. Traumatismos crânio-encefálicos. 